\title{
Construcción de una noción de aprendizaje para enfrentar la sociedad de la incertidumbre
}

Going towards a notion of learning to confront the society of uncertainty

\author{
Volumen 20, Número 2 \\ Mayo - Agosto \\ pp. 1-23
}

\section{Eduardo Carrasco Henríquez \\ Paola Quintanilla Godoy \\ Ramon Espinoza Salinas \\ Diego Escobar Riffo}

\section{Citar este documento según modelo APA}

Carrasco Henríquez, Eduardo., Quintanilla Godoy, Paola., Espinoza Salinas, Ramon. y Escobar Riffo, Diego. (2020). Construcción de una noción de aprendizaje para enfrentar la sociedad de la incertidumbre. Revista Actualidades Investigativas en Educación, 20(2), 1-23. Doi. 10.15517/aie.v20i2.41671 


\title{
Construcción de una noción de aprendizaje para enfrentar la sociedad de la incertidumbre Going towards a notion of learning to confront the society of uncertainty
}

\author{
Eduardo Carrasco Henríquez ${ }^{1}$ \\ Paola Quintanilla Godoy² \\ Ramon Espinoza Salinas ${ }^{3}$ \\ Diego Escobar Riffo 4
}

\begin{abstract}
Resumen: El objetivo de este artículo es mostrar los resultados de un proceso investigativo que se inicia en el año 2018 en la Universidad Metropolitana de Ciencias de la Educación (UMCE) en Santiago de Chile, y que tiene el propósito de resignificar la noción de aprendizaje en el marco de la formación inicial docente impartida en esta casa de estudios. Proceso que realiza un análisis bibliográfico, asociado a la descripción que hace Zygmunt Bauman de las sociedades líquidas. Para ello, se elabora un constructo de la noción de aprendizaje, que permita enfrentar con mejores herramientas, procesos de cambios e incertidumbre. El estudio tiene un enfoque cualitativo desde el cual se busca indagar la noción de aprendizaje a través de la selección de un corpus textual focalizado en torno al contexto y al uso de la palabra aprendizaje. Los hallazgos de la investigación muestran la caracterización de aprender mediante las siguientes dimensiones (i) aprendizaje como una experiencia, (ii) aprendizaje para un mundo en constante movimiento. En síntesis, es comprender la constante transformación que cada uno ha de vivir para estar en un mundo en constante e interdependiente evolución. De este modo, se espera que la noción de aprendizaje, para la sociedad de la incertidumbre, desarrolle un pensamiento dialógico, retroactivo, recursivo, hologramático que aborde la condición de autonomía y dependencia constante, es decir, que asuma la incertidumbre.
\end{abstract}

Palabras clave: aprendizaje, sociedad, formación docente.

Abstract: The goal of this article is to show the results of a research process that began in 2018, and that it has the purpose of re-signify the notion of education in the framework of the initial teacher training, considering the new requirements that the 21Th Century society puts to the educational system. These requirements are associated with the description of Bauman about the liquid societies; that's why we worked on the elaboration of a construct for the notion of learning for a society of uncertainty. The study has a qualitative focus, from which to investigate the notion of learning, through a focalized textual corpus, around the context and the use of the word learning. The findings of this investigation show the characterization of learning through the following dimensions, (i) learning as an experience, (ii) learning for a world in constant motion. In summary, the idea is to comprehend the constant transformation that each person has to live to be in a world in constant and interdependent evolution. In this way, the notion of learning for the society of uncertainty, it is expected to develop dialogical thinking, retroactive, recursive, hologrammatic that aboard the condition of autonomy and constant dependence, that assumes the uncertainty.

Key words: learning, society, teacher educators.

\begin{tabular}{l}
\hline Académico de la Universidad Metropolitana de Ciencias de la Educación, Chile, en el Departamento de \\
Educación Básica, Mención Matemática. Dirección electrónica: eduardo.carrasco@umce.cl
\end{tabular} Orcid https://orcid.org/0000-0002-9175-294X

${ }^{2}$ Académica de la Universidad Metropolitana de Ciencias de la Educación, Chile, en el Área Política y Gestión Educacional, Departamento de Formación pedagógica. Dirección electrónica: paola.quintanilla@umce.cl Orcid https://orcid.org/0000-0003-4991-8392

3 Académico de la Universidad Metropolitana de Ciencias de la Educación, Chile, en el Área Gestión y Convivencia Escolar, Departamento de Artes Visuales. Dirección electrónica: ramon.espinoza@umce.cl Orcid https://orcid. org/0000-0003-4658-8498

${ }^{4}$ Sociólogo, Metodólogo, Chile. Dirección electrónica: descobar13@gmail.com Orcid https://orcid.org/0000$\underline{0001-8812-1736}$

Artículo recibido: 13 de octubre, 2019

Enviado a corrección: 20 de febrero, 2020

Aprobado: 27 de abril, 2020 


\section{Introducción}

Diversos autores están hablando de nuestra sociedad actual como una sociedad donde el cambio y la incertidumbre son características centrales. El cambio climático, los cambios políticos y sociales, y la cada vez mayor conectividad hacen que las certezas del pasado, tales como la nacionalidad, las formas de relacionarse y la escuela como espacio central de aprendizaje, se desdibujen a la mirada educativa. Ya no basta con la discusión sobre qué elementos configuran un curriculum y/o cuáles han de propiciar la práctica educativa, sino que es necesario repensar aquellas palabras que nos definen como docentes, esos conceptos construidos en un mundo de certezas, que hoy al decir de Baumam (2005), se licúan. En este marco, el presente artículo, es el resultado de una investigación que busca identificar la brecha entre la o las nociones de aprendizaje que sustentan la acción educativa en formadores y formadoras de docentes, y la noción de aprendizaje que requiere una educación pertinente a los desafíos futuros del estudiantado, así como avanzar en actualizar la formación de docentes con respecto a los desafíos de esta sociedad de la incertidumbre.

Esta discusión sobre los desafíos se hace necesaria cuando reconocemos como esencial la capacidad de actuar del profesorado, para el logro de los aprendizajes, para tomar decisiones y elecciones, así como para asumir una posición en relación con su trabajo e identidad profesional, (Eteläpelto, Vähäsantanen, Hökkä, y Paloniemi, 2013; Ketelaar, Beijaard, Boshuizen, y Den Brok, 2012; Lasky, 2005; Lipponen y Kumpulainen, 2011). Decisiones pedagógicas en que el significado de aprendizaje, como noción construida en tiempos de una sociedad sólida, está en la base de los marcos orientadores del actuar docente. Esto configura un problema, dado que las y los docentes y las escuelas aún mantienen un rol social y político movilizador y transformador. No obstante, el aula, comprendida como aquel salón amurallado, generalmente de grandes ventanas que alberga un grupo numeroso de estudiantes de edades similares y en que el profesorado representa el liderazgo institucional y la potestad del saber aprendible, es uno de los espacios que se ha mostrado más poroso en el último tiempo.

En la actualidad, el espacio del aula se ha visto permeado; por una parte, por la constante vigilancia social, la cual pareciera preocuparse más por los indicadores internacionales, en relación con los resultados estandarizados de aprendizaje que constituyen ranking entre la comunidad escolar. Este fenómeno de medición ha aumentado el número de actores que busca incidir en los procesos educativos, debilitando el liderazgo académico del profesorado. También el hecho de convivir con la cultura digital a través de 
internet y las redes sociales que ofrecen inmediatez y acceso a la información, abriendo espacios no formales de aprendizajes, lo que ha ido mermando el rol tradicional del docente, así como la legitimidad de la escuela en cuanto a su centralidad respecto de los saberes que han de ser aprendidos. La escuela, y en particular, el profesorado como primer responsable y actor visible del acto educativo, son demandados desde la sociedad a una mejor educación. Sin embargo, esta idea de mejor educación, mediada por las evaluaciones estandarizadas, pareciera invisibilizar necesidades educativas locales para colocar en la agenda de políticas educativas nociones y estándares globalizados respecto del aprendizaje.

Por lo mismo, cuando queremos referirnos al aprendizaje, desde los contextos pedagógicos, es inevitable que hablemos de la escuela y de las prácticas que en ella se reproducen. Son pocas las escuelas de pedagogía que en los procesos formativos realizan la reflexión de cómo el estudiantado es sujeto activo de su aprendizaje. Existen algunos esfuerzos en esta área que orientan a que los estudiantes de pedagogía no solo logren aprender a aprender, sino, al mismo tiempo, aprender a enseñar a otros a aprender de modo autónomo. Esto, sin reducir este acto a un aprendizaje disciplinario específico, se trata más bien de una experiencia transdisciplinar.

La formación inicial docente no es capaz de transformar el contrato de la clase tradicional y el orden activo-pasivo de la jerarquía docente-estudiante, en el cual, el profesor coloca el saber en el aula y el estudiante debe llegar a ese saber lo más similar posible a eso que el profesor enseña. Entendiendo que dichas transformaciones incidirán directamente no solo sobre las operaciones y prácticas de aprendizaje de las y los estudiantes, sino que también, y al mismo tiempo, sobre las operaciones y prácticas de enseñanza que esos mismos estudiantes deberán, como profesores y profesoras, ser capaces de instaurar y promover al momento de su ejercicio docente. (Collingwood-Selby, 2018,).

Creemos que el estudiantado de pedagogía no derriba las construcciones que hace desde su propia experiencia escolar. Cuando vive su formación en las aulas universitarias. Es decir, los procesos formativos de las escuelas de pedagógica no son suficientes para desbaratar las concepciones tradicionales de aquella sociedad sólida que configuró la escuela del siglo XX. Por ello, en los esfuerzos de cambio, las visiones profesionalespersonales son un factor relevante para determinar cuándo y cómo el profesorado y otros miembros claves de la comunidad escolar realizan esfuerzos para la transformación (Hammerness, 2010). 
Esta dicotomía entre las experiencias que viven los futuros profesores en los espacios formativos y los tiempos de incertidumbre que experimenta la sociedad, impone la urgente necesidad de repensar la noción de aprendizaje que está en la base de la experiencia formativa en las escuelas de pedagogía. Considerando que, en la sociedad actual, las certezas del pasado se desdibujan, licuan, ante procesos complejos (en el sentido de Morin) de cambios y dudas, que se imponen a los sistemas educativos, por lo tanto es preciso generar un diálogo entre las nociones de aprendizaje de los formadores de formadores y sus prácticas, en las cuales se consideren los nuevos escenarios de la sociedad líquida (Bauman, 2007) y las nuevas estrategias que se implementen para enfrentar dichos cambios.

El primer paso que hemos querido dar es poder reconocer la noción o nociones de aprendizaje que emergen en una sociedad de la incertidumbre a través de revisión bibliográfica, para avanzar en la resignificación de las palabras que permiten explicar, argumentar y proyectar nuestra acción educativa. En particular, la palabra aprendizaje, que está en la base del discurso, así como de la acción pedagógica, no solo es un objeto técnico para la descripción de lo que un estudiante conoce, sino que es parte y conforma un lenguaje que, como señala Guerrero (2012), es un instrumento que transmite sentimientos, ideas, conceptos y juicios.

De modo preciso, este artículo presenta el constructo de aprendizaje para la sociedad de la incertidumbre, y se propone como base, caracterizar la brecha entre la noción de aprendizaje de profesorado formador, con esta nueva noción de aprendizaje para la sociedad de la incertidumbre y, de esta forma, hacer posible estructurar y proponer los cambios necesarios para las escuelas de pedagogía.

Levantar un constructo de aprendizaje fue un trabajo basado en una metodología interpretativa comprensiva, desarrollada durante los años 2017 y 2018 en la Universidad Metropolitana de Ciencias de la Educación (UMCE) en Santiago de Chile, financiado por fondos estatales asignados a las instituciones de educación superior en el marco de sus Planes de Mejoramiento Institucional (PMI) PNII-04.2017. 


\section{Referente Teórico}

En los últimos años los cambios sociales se han caracterizado por ser sorpresivos, vertiginosos y por superar las estructuras sociales y políticas que no se encuentran preparadas para dichos cambios, una de esas estructuras a nuestro parecer es la escuela. Las redes sociales, con soporte en la internet y con mayor conectividad de los ciudadanos planetarios, está fortaleciendo los procesos de intercambio cultural a la vez transforma a cada sujeto conectado en un potencial proveedor de contenido informacional. Lo anterior, es una de las razones por las cuales Bauman (2005) propone caracterizar la sociedad como una sociedad líquida. Bajo esta metáfora Bauman expone procesos de porosidad en los cuales las certezas y la solidez que caracterizaron el siglo XX pierden su vieja rigidez y por lo tanto permiten que lo social muestre su impredecibilidad. Es una sociedad en que el cambio es central, en que lo permanente se desvaloriza, igual que el conocimiento a construir, entonces, la educación de la escuela ya no es un espacio para construir aquel núcleo duradero del cual dependa el fututo, como señala Bauman.

(...) el cambio actual no es como los cambios del pasado. En ningún otro punto de inflexión de la historia humana los educadores debieron afrontar un desafío estrictamente comparable con el que nos presenta la divisoria de aguas contemporánea. Sencillamente, nunca antes, estuvimos en una situación semejante. Aún debemos aprender el arte de vivir en un mundo sobresaturado de información. Y también debemos aprender el aún más difícil arte de preparar a las próximas generaciones para vivir en semejante mundo. (Bauman, 2005, p. 25)

Por su parte Morin, pone en evidencia la necesidad de una nueva mirada a nuestro mundo, es una mirada desde la complejidad, que se entiende como aquel entretejido que no se puede separar. Sin embargo, reconoce la actual imposibilidad de mirar el todo, sus acciones y retroacciones. Es decir, la imposibilidad de un dispositivo que nos permita separar ilusión de realidad, conocimiento de fantasía. Es, por el contrario, la falibilidad del saber que tenemos una constante, todo saber es posible de ser refutado, dejar su rango de validez para tener que ser reconstruido o reemplazado. El hecho de que el sistema solar pasara en los años noventa de tener nueve planetas a solo ocho, es una muestra de que incluso las verdades científicas son propicias de cambiar. Entonces, es necesario "mantener una incertidumbre racional: si no mantiene su vigilante autocrítica, la racionalidad se arriesga 
permanentemente a caer en la ilusión racionalizadora; es decir que la verdadera racionalidad no es solamente teórica ni crítica sino también autocrítica" (Morin, 1999, p.8).

Entonces debemos prepararnos para lo inesperado, y que hoy sorprende toda vez que hemos instalado y asignado la seguridad de las teorías, sobre todo científicas, como verdades perdurables en un universo, material y social, que hoy sabemos están en constante movimiento. De este modo, entendemos como sociedad de la incertidumbre a esta época, en que lo central de la vida es el cambio constante e impredecible. Esta definición, no pretende ser una categoría sociológica de análisis, sino que su alcance en este artículo se reduce a una definición operacional de nuestra investigación.

A partir de lo anterior, tanto Bauman como Morin plantean una preocupación con la educación para la incertidumbre que ha de ser vivida. Cada uno de ellos aborda, desde sus perspectivas, la necesidad de formación de un nuevo sujeto que, para Morin, se dará a partir de la reforma de la consciencia, y para Bauman, desde la formación de las identidades, que concurren a todo instante de forma no fragmentadas, superando la idea de que en la diferencia del objeto esta la imposición de una(s) identidad(es) en relación con otras (Câmara, Fonseca y Souza, 2014).

Los estudios sobre docentes han ido reconociendo el rol de continuos elaboradores a partir de lo cual las y los docentes juegan un papel activo en la transformación del conocimiento para ser enseñado. Por tanto, es necesario avanzar en el cambio de las visiones profesionales personales, para determinar cuándo y cómo el profesorado -y otros miembros clave de la comunidad escolar- realizan esfuerzos para el cambio (Hammerness, 2010). Es necesario reconocer que el actuar del formador de docentes se constituye a partir de las nociones y experiencias que le forman y que pueden variar desde una noción de aprendizaje como transferencia de contenidos a un aprendizaje como construcción colectiva de conocimiento. (Reyes, Jedlicki, Miranda, Santa Cruz, Cornejo, Núñez, Arévalo e Hidalgo. 2014). Luego, los espacios de formación de docentes se constituyen en un primer espacio de cambio, por tanto, los formadores del profesorado son quienes deben liderar los procesos de transformación.

\section{Metodología}

\subsection{Enfoque}

Se asume un enfoque teórico cualitativo, en cuanto se busca indagar respecto de la noción de aprendizaje dando sentido o interpretando los fenómenos en los términos del 
significado que las personas de la comunidad les otorgan (Vasilachis de Gialdino, 2006). Se asume este enfoque, pues permite modelar un proceso inductivo, contextualizado y que en el proceso de recolección de datos o levantamiento de información incluye también las percepciones, experiencias e ideologías de las y los investigadores, enriqueciendo así este proceso. El objetivo final de este tipo de estudio es comprender fenómenos complejos o nuevos.

\subsection{Unidades de Análisis}

La primera parte de la investigación se centró en el análisis bibliográfico para la definición del constructo de aprendizaje y la sociedad de la incertidumbre. Se revisan diversos autores que se refieren a la sociedad actual, reconociéndola en un estado de alta complejidad e incertidumbre. Además, se incorporó bibliografía de modelos de enseñanza y aprendizaje, tales como: el aprendizaje autorregulado, constructivismo, cognitivismo y las Neurociencias. Durante un período de 5 meses $^{5}$ los integrantes del equipo de investigación revisaron material bibliográfico referido a los temas mencionados y seleccionaron una serie de referencias (libros, ensayos, artículos) que luego sistematizaron en fichas bibliográficas. La selección final contó con un total de 19 referencias: 5 ensayos, 4 libros de difusión, 4 reportes de investigación y 6 revisiones bibliográficas (Arriagada, Ibañez y Godoy, 2019).

A partir de esta sistematización, se realiza la construcción de nodos de sentido, y mediante la aplicación de una técnica de análisis de contenido (Espín, 2002) se avanzó en la caracterización de aspectos que concurren a comprender el aprendizaje desde la bibliografía revisada. Dicho trabajo estuvo fuertemente marcado por la intencionalidad del equipo investigador y de los marcos teóricos iniciales descritos en el apartado anterior.

Luego, considerando las fichas bibliográficas y la primera sistematización de textos y publicaciones en torno a la noción de aprendizaje, la búsqueda se centró en la selección de unidades textuales conformadas por párrafos que contienen palabras clave o sinónimos de estas, con el objetivo de conformar un corpus de análisis, que permitió levantar codificaciones semánticas, para la construcción de los significados que emergen en la producción académica en torno a la sociedad de la incertidumbre. De esta forma, las fichas de sistematización bibliográfica se sometieron a un análisis con el software $R$, versión 3.5.0,

\footnotetext{
${ }^{5}$ Esta etapa de revisión y sistematización bibliográfica de la investigación se desarrolló entre los meses de agosto y diciembre de 2017.
} 
mediante la librería $R Q D A$ y $\mathrm{tm}^{6}$, para la construcción de nodos de sentido o semánticos. Estos análisis permitieron identificar patrones y establecer un orden (Pulido y Rodríguez, 2014) en las fichas analizadas. También, facilitó la organización por categorías, de forma exploratoria, en las cuales, los elementos discursivos subyacentes en la bibliografía analizada permiten comprender el lugar del aprendizaje para una sociedad de la incertidumbre que sustentan las y los autores de los textos seleccionados.

El corpus de análisis, que da cuenta de las palabras claves y que permiten levantar codificaciones semánticas, emerge de la bibliografía y de la producción académica en torno a temas relacionados con la investigación, tales como; la sociedad de la incertidumbre, concepciones y descripciones de tipos de aprendizaje pertinentes al tipo de sociedad en la que vivimos, formación inicial docente. Al interior de estos temas se seleccionaron párrafos como unidades textuales, que contienen palabras claves que dan cuenta de la visión de los autores respecto a los temas referidos. De modo particular, el primer criterio de inclusión de los textos fue la declaración de la sociedad líquida o de la complejidad en el título o en el enfoque teórico. Un segundo criterio de inclusión fue la selección de textos y publicaciones relacionadas con modelos de enseñanza tradicionales, como aprendizaje autorregulado, constructivismo, cognitivismo y aportes de las neurociencias. En este ejercicio, no se incluyeron párrafos que se refirieran a otros tipos de sociedad, concepciones más tradicionales de aprendizaje y formación de otro tipo de personas distintas a los y a las docentes.

\subsection{Técnicas de Recolección}

La revisión bibliográfica inicial realizada por el equipo investigador, permitió elaborar una "Matriz Contenedora" que identificó: la fecha de revisión inicial, el integrante del equipo que realizó la revisión, el tipo de texto (ensayo, libro, revisión bibliográfica o libro de difusión), el título del texto, el autor, las palabras claves identificadas por el investigador que realizó la revisión, y el aporte identificado para comprender la noción de aprendizaje del autor ("Aprender es"), el contexto en que se realiza el aprendizaje y el sentido de aprender ("se aprende para") (Arriagada, Ibañez y Godoy, 2019). Posteriormente, se seleccionaron 19 textos para ser analizados. El primer criterio de inclusión de los textos fue la declaración de

${ }^{6}$ RDQA es un paquete del software R para análisis de datos cualitativos gratuito, que funciona en las plataformas Windows, Linux y Mac OSX. RQDA es una herramienta fácil de usar para ayudar en el análisis de datos textuales e incluye una serie de características estándar de análisis de datos cualitativos asistidos por computadora. Hasta cierto punto, RQDA y $\mathrm{R}$ hacen una plataforma integrada para el análisis de datos cuantitativos y cualitativos. 
la sociedad líquida y/o complejidad en el título o bien, en el enfoque teórico. Un segundo criterio de inclusión fue la búsqueda por académicos líderes de corriente educativas. La elaboración de las fichas de sistematización identifica palabras claves, el contexto en el cual se puede situar el contenido del documento revisado, comentarios y párrafos textuales de los aspectos que permiten definir, tanto la noción de aprendizaje, como "el para qué se aprende" en el texto seleccionado. A partir de ellos avanzar en la descripción de las nociones en torno a aprender que se encuentran presentes en diferentes corrientes pedagógicas, para dar cuenta del tipo de aprendizaje que se requiere para la sociedad de la incertidumbre. Con esta revisión interpretativa y comprensiva se elaboró una "Tabla de operacionalización de variables" para establecer, desde las Corrientes Pedagógicas, cómo se entiende el proceso aprender (“Aprender es”), su relación con el contexto y el sentido de dicho aprendizaje ("Aprender para"), lo que dio forma al corpus de análisis configurado por unidades textuales de la bibliografía sistematizada.

El corpus de análisis seleccionado se sometió a dos procedimientos propios del proceso metodológico de la Teoría Fundamentada: el ajuste y el funcionamiento (BonillaGarcía y López-Suárez, 2016). El ajuste es la generación de categorías conceptuales a partir de los datos, y el funcionamiento es la capacidad de las categorías conceptuales para explicar lo investigado. El proceso de recolección permitió identificar los atributos, cualidades o características de las unidades textuales o párrafos, seleccionados para establecer coincidencias y diferencias. Aquellas unidades que compartían las mismas características fueron codificadas o categorizadas con un mismo rótulo o nombre que permitió identificarlas con el concepto al que pertenecían dentro del tema de investigación correspondiente.

\subsection{Procesamiento de análisis}

La elaboración del constructo de aprendizaje para la sociedad de la incertidumbre se realizó a partir de la selección de un corpus textual focalizado, conformado a través de la selección de textualidades emergidas en los textos escogidos.

El análisis bibliográfico se abordó desde dos miradas metodológicas complementarias. La primera contempló la construcción de fichas bibliográficas que reportan la visión de cada persona autora. Desde el lugar del currículum y pensando en el aprendizaje se orienta el primer acercamiento a los textos escogidos, desde tres categorías iniciales: "aprender es", "aprender para" y el "contexto de aprendizaje" evidenciados en cada texto. Estas categorías emergen del propósito del proyecto y se inspiran en categorías basales del curriculum 
tradicional, que se pregunta, ¿cómo hacer?, ¿a quienes?, ¿con qué resultados?, desde el lugar del currículum y pensando en el aprendizaje se orienta el primer acercamiento a los textos escogidos, desde dos categorías: "aprender es", "aprender para" las que actúan como frases orientadoras para la descripción.

El análisis de las fichas se realizó a partir del levantamiento de nodos de sentido y de categorías para el análisis del contenido. Esto permite avanzar en la interpretación del discurso subyacente en los textos, logrando una caracterización de los aspectos que conforman la noción o nociones de aprendizaje presentes en los textos.

La segunda mirada se estructuró a partir del análisis cualitativo de los datos o unidades textuales (párrafos) seleccionados por medio del software $R+$, versión 3.5.0 (librería RQDA), lo que dejo identificar las palabras utilizadas con mayor frecuencia y, a partir de ellas, seleccionar los párrafos más relevantes para realizar el análisis semántico. Esto permitió generar redes de sentido, es decir la construcción de mapas de topología y medición, que expresan la emergencia de descripciones, de cogniciones relevantes epistemológicamente, utilizando planteamientos teóricos que combinan planteamientos estructurales constructivistas y sistémicos. Los datos que compartían las mismas características se agruparon y se les asignó un rótulo o un nombre determinado que indica el concepto al que pertenecen. Es decir, se congregan en un mismo código (según el nombre que reciben en el software de análisis cualitativo) o subcategoría, según correspondía (Jiménez-Fontana, García-González, Azcárate, Navarrete y Cardeñoso, 2016). Este tipo de análisis de contenido permitió avanzar en la interpretación comprensiva del discurso que contenían las unidades textuales. Para esto, el equipo de investigación, en reuniones de trabajo, revisó de forma permanente para ajustar el corpus de análisis y las redes de sentido a los objetivos de la investigación.

\section{Resultados}

En una sociedad de la incertidumbre, entonces, el aprender ha de concebirse líquido, en cuanto a que aquello que se aprende puede ser necesario desaprenderlo o modificarlo. Al decir de Bauman (2007), lo imperecedero, ese aprender para toda la vida, no tiene sentido. O en un contexto de complejidad el aprender debe ser siempre entendido desde la falibilidad de un saber que evoluciona y que puede perfectamente hacerse inútil. Del mismo modo, el "para qué aprender" emerge desde aquella sociedad que le da un uso a dicho aprendizaje, así como desde las prácticas específicas de los distintos actores sociales. Por ejemplo, el 
aprendizaje promovido por los estándares internacionales en el marco de OCDE ha modificado la forma de aprender la literatura, supeditándola al lenguaje y a la comunicación, en tanto se reconocen como herramientas necesarias para el desarrollo de las economías. Luego, más que construir una noción de aprendizaje, el constructo asume una red de nociones en torno a los focos descritos que configuran el aprendizaje

A partir de tres focos iniciales definidos para la construcción de las fichas analíticas: "Aprender es", "aprender para" y "contexto", se construyó la Tabla 1, que permite la operacionalización de variables con revisiones bibliográficas al identificar las palabras y conceptos más repetidos en las revisiones del equipo investigador. 
Tabla 1. Conceptos más frecuentes encontrados en la revisión bibliográfica

\begin{tabular}{|c|c|c|c|c|c|c|c|c|}
\hline & \multicolumn{5}{|c|}{ Artículos referidos Sociedad Liquida y Complejidad } & \multicolumn{3}{|c|}{ Artículos referidos a Corrientes pedagógicas } \\
\hline \multirow{5}{*}{ 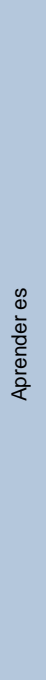 } & $\begin{array}{l}\text { Mundo en } \\
\text { constante } \\
\text { movimiento } \\
\text { experiencial }\end{array}$ & $\begin{array}{l}\text { Tecnología y } \\
\text { millenials } \\
\text { Exploración }\end{array}$ & $\begin{array}{c}\begin{array}{c}\text { Cognición del } \\
\text { estudiante }\end{array} \\
\text { Transmisión hábitos }\end{array}$ & $\begin{array}{l}\text { Comunidad de } \\
\text { aprendizaje } \\
\text { Construcción de } \\
\text { conocimiento colectivo }\end{array}$ & \begin{tabular}{|c|} 
El aprendizaje \\
autorregulado \\
Proceso \\
proactivo/motivación
\end{tabular} & $\begin{array}{c}\text { Constructivismo } \\
\text { social }\end{array}$ & $\begin{array}{l}\text { Neurociencias } \\
\text { Proceso } \\
\text { humano }\end{array}$ & $\begin{array}{l}\text { Cognitivismo } \\
\text { Acceder al objeto }\end{array}$ \\
\hline & resistencia & Múltiples & Transmisión valores & $\begin{array}{l}\text { Construcción de } \\
\text { conocimiento colectivo }\end{array}$ & Destreza y voluntad & Sentimiento & $\begin{array}{l}\text { Base a la } \\
\text { corporalidad }\end{array}$ & $\begin{array}{c}\text { Sistema de } \\
\text { Representación }\end{array}$ \\
\hline & Acción critica & $\begin{array}{l}\text { Formas } \\
\text { propias }\end{array}$ & $\begin{array}{l}\text { Transmisión } \\
\text { actitudes }\end{array}$ & Equipos & Crítico & Imaginar & Experiencial & $\begin{array}{l}\text { Capacidad de } \\
\text { representar } \\
\text { aquello que se } \\
\text { conoce }\end{array}$ \\
\hline & Transformar & Secuencial & Reproducción & Flexibilidad & $\begin{array}{l}\text { Construcción } \\
\text { Saber/cognición }\end{array}$ & Recordar & Contexto social & $\begin{array}{l}\text { Aprender de } \\
\text { manera activa }\end{array}$ \\
\hline & & exploración & Replicación & $\begin{array}{c}\text { Nuevas } \\
\text { configuraciones } \\
\text { sociales }\end{array}$ & Conciencia & $\begin{array}{c}\text { Construir nuevo } \\
\text { conocimiento }\end{array}$ & Cultural & Observación \\
\hline \multirow{6}{*}{ 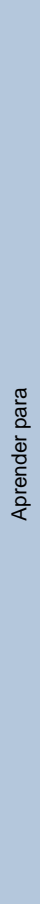 } & $\begin{array}{l}\text { Desaprender } \\
\text { Resiste la } \\
\text { incertidumbre }\end{array}$ & $\begin{array}{l}\text { Información } \\
\text { Web } 2.0 \\
\text { Desafios }\end{array}$ & Vivir & $\begin{array}{c}\text { Nuevas prácticas } \\
\text { Transformar }\end{array}$ & $\begin{array}{l}\text { Toma Conciencia } \\
\text { Competencias en } \\
\text { inventores de } \\
\text { inteligencia }\end{array}$ & $\begin{array}{l}\text { Cohesión } \\
\text { Visión sistémica } \\
\text { de elementos }\end{array}$ & $\begin{array}{l}\text { Visión Crítica } \\
\text { de la sociedad } \\
\text { Visión crítica de } \\
\text { la educación }\end{array}$ & $\begin{array}{c}\text { Solución que se } \\
\text { busca } \\
\text { Cambiar } \\
\text { representaciones } \\
\text { para resolver } \\
\text { problemas }\end{array}$ \\
\hline & $\begin{array}{l}\text { Complejidad } \\
\text { del mundo }\end{array}$ & Mundo digital & Revolución cognitiva & $\begin{array}{l}\text { Construcción de } \\
\text { sentido }\end{array}$ & Metas & Formación & & \\
\hline & Acción crítica & \begin{tabular}{|c|} 
Entornos \\
personales de \\
aprendizaje
\end{tabular} & Aprendizaje & $\begin{array}{c}\text { Participación/experticia } \\
\text { adaptativa }\end{array}$ & Educación superior & Educación & & \\
\hline & $\begin{array}{l}\text { Resolver } \\
\text { problemas }\end{array}$ & \begin{tabular}{|c|} 
Redes \\
personales de \\
aprendizaje
\end{tabular} & $\begin{array}{l}\text { Meta complejidad } \\
\text { educativa }\end{array}$ & Cultura del simulacro & & Familia & & \\
\hline & $\begin{array}{l}\text { Sociedad } \\
\text { cambiante }\end{array}$ & $\begin{array}{c}\text { Tic en } \\
\text { educación }\end{array}$ & Bucle educativo & Diálogo analítico & & sociedad & & \\
\hline & $\begin{array}{l}\text { Sujeto nómade } \\
\text { Complejidad } \\
\text { mundo }\end{array}$ & & \begin{tabular}{|c} 
Enfoque \\
sistémico/aprendizaje \\
Reaprendizaje
\end{tabular} & & & $\begin{array}{l}\text { Optimizar } \\
\text { rendimiento } \\
\text { Promover } \\
\text { procesos } \\
\text { dialógicos }\end{array}$ & & \\
\hline \multirow{4}{*}{ 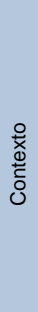 } & Incertidumbre & Exploración & & & & & & \\
\hline & $\begin{array}{l}\text { Revolución } \\
\text { Científica }\end{array}$ & & & & & & & \\
\hline & $\begin{array}{l}\text { Complejidad } \\
\text { del mundo }\end{array}$ & & & & & & & \\
\hline & $\begin{array}{l}\text { Contextos } \\
\text { diversos }\end{array}$ & & & & & & & \\
\hline
\end{tabular}

Fuente: Arriagada, Ibañez y Godoy, 2019, p. 231. (Sic) 
De esta forma, la revisión permitió dar cuenta, en primer término, del carácter transversal del contexto respecto de los otros dos focos. Luego, la red de nociones que configuran la concepción de aprendizaje para la sociedad de la incertidumbre, que emergen de las lecturas, está siempre imbricada al contexto en que ese aprender es reflexionado o propuesto. Se asume que el aprendizaje es un proceso estrictamente social, a través del cual se vinculan las herramientas y los signos culturales. Un proceso en el cual estudiantes y docentes son entendidos como seres sociales productos de interacciones socio-culturales de las que son parte. Así, es un aprender en y para un contexto.

En segundo término, se construyen "nodos de sentido" que permiten la caracterización de la red de nociones de aprendizaje. A saber:

- El mundo está en constante movimiento. Esto es un mundo que cambia y que genera incertidumbre.

- Tecnología y milenial. Como nodo desde el cual se comprende que las y los estudiantes viven en un nuevo milenio y altamente conectado con las tecnologías.

- La cognición del estudiante. Qué diversos aportes han ido develando los procesos de aprendizaje, que transita de un aprender memorístico (memoria semántica) a un aprender distinto (memoria de trabajo).

- La noción de comunidad de aprendizaje, que reconoce el carácter colectivo del aprendizaje en comunidades de aprendizaje.

La segunda mirada parte de la identificación de palabras relevantes. Usando software $\mathrm{R}$ se construye la Figura 1, de palabras más usadas. 
Figura 1. Nube de Palabras, corpus textual de investigación.

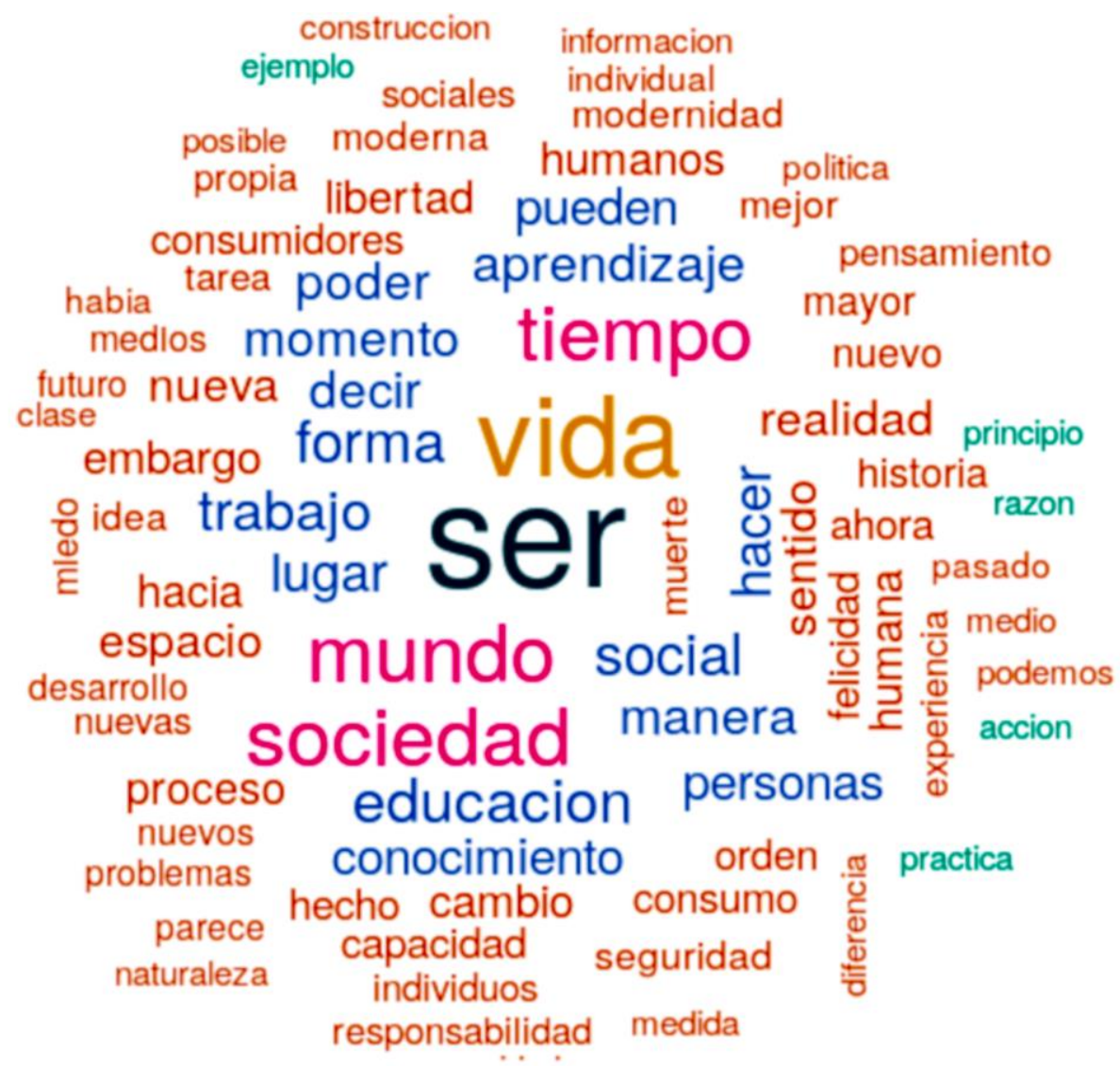

Fuente: elaboración propia, 2019

Esto permitió una búsqueda de párrafos asociados a dichas palabras junto con la palabra aprendizaje. En un proceso de codificación, de las textualidades extraídas, se clasifican los nodos de sentido que emergieron del análisis anterior, pero se avanza en una red de asociaciones a elementos que caracterizan dichos nodos (Ejemplo figura 2).

Esto permitió una búsqueda de párrafos asociados a dichas palabras junto con la palabra aprendizaje. En un proceso de codificación, las textualidades se clasifican en los distintos nodos de sentido emergidos del análisis anterior, y se avanza en una red de asociaciones de elementos que caracterizan dichos nodos (Ejemplo figura 2). 
Figura 2. Esquema de sentidos relacionado al nodo "Mundo en Constante Movimiento".

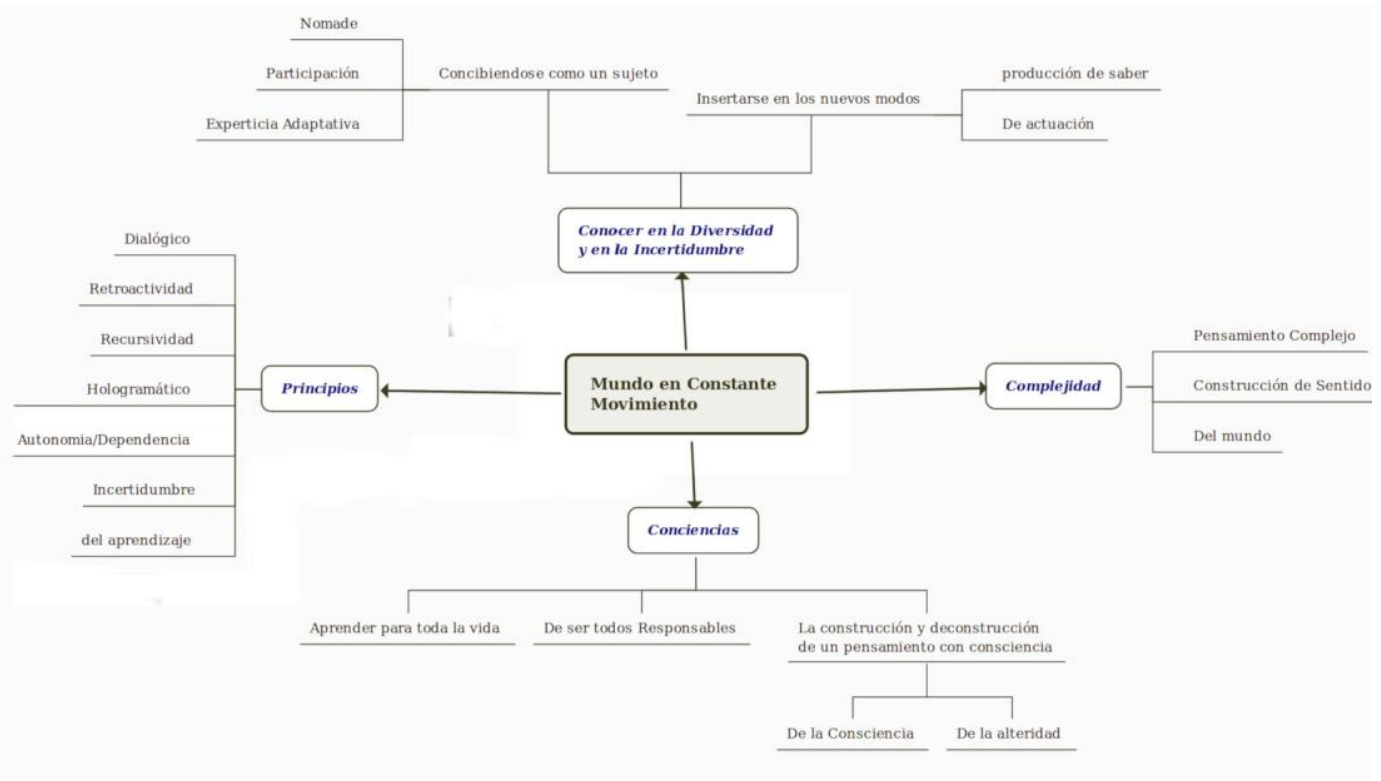

Fuente: Elaboración propia, 2019.

A partir del esquema, "mundo en constante movimiento", se realizó una operacionalización de las definiciones teóricas de los nodos de sentido. Asociando conceptualmente las textualidades a dichos nodos, los que permiten identificar las variables que posibilitan realizar una medición de las nociones de aprendizaje que concurren a la toma de decisiones didácticas en los formadores de formadores. De esta forma, los nodos relevantes se constituyen en dimensiones de la noción de aprendizaje, cada una de las cuales se caracteriza en un conjunto de variables medibles.

Las perspectivas constructivistas asumen que la construcción de aprendizaje se basa en la actividad que realiza el estudiantado. Luego en una visión compleja si bien se reconoce que quien es estudiante es un constructor activo de su conocimiento, esta construcción es desde el contexto que le toca vivir (Flores, 2011). Entonces, la práctica se constituye como componente fundamental en el proceso educativo. No hay verdadero aprendizaje si no se hace una práctica para el aprendizaje (Armijos, Hernández y Sánchez, 2017). Sin embargo, aprender no ha de ser un proceso de acumular saberes, sino que, ha de ser un proceso en que las personas sean capaces de hacer cosas nuevas, no necesariamente repetir lo que han hecho otras generaciones. Es decir, que sean creativas, inventivas y descubridores capaces de crear una actitud para aprender a actuar en contextos complejos que van más allá de su disciplina de estudio y para que aborden aspectos de la vida misma. (Flores, 
2011). Así uno de los elementos más simples en torno al aprendizaje es que la practica incrementa el aprendizaje: en el cerebro, hay una relación similar entre la cantidad de experiencia en un ambiente complejo y el monto de cambio estructural (Posner y Rothbart, 2005, De la Barrera y Donolo, 2009). Hay evidencias que señalan que tanto un cerebro en desarrollo como uno ya maduro se alteran estructuralmente cuando ocurren los aprendizajes (Bransford, Brown, Cocking, 2003, De la Barrera y Donolo, 2009). Además, el aprendizaje como una actividad situada en un contexto que da significado al proceso. Toda adquisición de conocimiento se contextualiza en algún tipo de actividad social, (De Oliveira et al. 2015) es un proceso que esta contextualizado en una comunidad, con su sistema de valores, metas y dinámicas de participación.

Iniciamos, con conceptos que permitieron establecer nodos de sentido que iban agrupando dichos conceptos, para luego proveer de textualidades a los nodos, evaluando su pertinencia, estos comienzan a perfilarse como las dimensiones de la noción de aprendizaje, una noción que se construye en la imbricación de los nodos, los que va constituyendo el espacio de significado de aprendizaje, más que una definición dada.

Ejemplo de ello, la situación de Tecnología y Millenials, se espera que las metas educativas se construyan en función de lo que la cultura considera valioso. Es una construcción colectiva en la sociedad de la incertidumbre, por lo tanto, son valiosas las TIC, que ofrece espacios de nueva información en mundo digital de redes personales de aprendizaje (digitales, presenciales). Por su parte la cognición del estudiante, desarrolla la posibilidad de cambiar para afrontar la incertidumbre y la complejidad que son los signos esenciales de estos tiempos. Es central entonces el desaprender, que no es más que dejarnos permear por lo imprevisto y lo incierto. El devenir del sujeto es el desaprendizaje de saberes disimiles, divergentes que están en constante construcción y deconstrucción a partir del cuestionamiento constante y la autorregulación consciente. Es una revolución científica, es tomar consciencia que somos movimiento, devenir, creación, descubrimiento permanente. De la interdependencia entre observador y mundo. Es desvelar el sentido del sí mismo en relación con los otros y la red de procesos de aprendizaje que a lo largo de la vida conducen a observar de una manera particular la realidad. Es buscar la realización personal en franca apertura al desarrollo social y en equilibrio con el medio ambiente es el cambio constante de la estructura neuronal. Es el desarrollo de nuevas vías neurales, nuestras sinapsis cambian todo el tiempo. Es un incremento constante desde la práctica que se vive. Aprender no es llenar de datos al estudiante. La memoria es potencialmente inhabilitante, engañosa e inútil. 
En cuanto a las Comunidades de Aprendizaje, se destaca la construcción colectiva como central y, dado que hay una generación de nuevas configuraciones sociales, se requiere enriquecer el conocimiento de las partes al todo y del todo a las partes en un mismo movimiento productor de aprendizajes. Finalmente, los entornos personales de aprendizaje (PLE), promueven que el individuo coloque sus conocimientos y competencias en vista de la construcción de un ethos que impacta significativamente en la vida social, económica y medioambiental de los miembros de la comunidad.

Así, las variables definidas para cada una de las dimensiones identificadas se resumen en la Tabla 2, siguiente:

Tabla 2. Dimensiones y variables seleccionadas para "Aprender Es"

\begin{tabular}{|l|l|}
\hline Dimensión & Variable \\
\hline \multirow{2}{*}{$\begin{array}{l}\text { El Aprendizaje como una } \\
\text { Experiencia }\end{array}$} & El aprendizaje como producción \\
\cline { 2 - 2 } & El aprendizaje es una acción \\
\cline { 2 - 2 } Cognición del Estudiante & $\begin{array}{l}\text { El aprendizaje como proceso social } \\
\text { Uso del lenguaje para relacionarse y transmitir } \\
\text { formación }\end{array}$ \\
\cline { 2 - 2 } & \begin{tabular}{l} 
Uso de estrategias u operaciones mentales \\
\cline { 2 - 2 } Comunidades de Aprendizaje
\end{tabular} \\
\cline { 2 - 2 } & Procesamiento de información \\
\hline \multirow{2}{*}{ Tecnología y Millenials } & Espacios personales \\
\hline
\end{tabular}

Fuente: Elaboración propia (2019), a partir del análisis de contenido de la bibliografía.

El mismo análisis se realizó en el segundo foco de atención, a saber, el Aprender para. En este foco, en el nodo de mundo en constante movimiento, emerge la concepción de un mundo líquido que cambia su forma y horada lo seguro de siglos pasados en la cultura occidental. Colocando la incertidumbre como aspecto siempre presente y que por tanto se requiere abordar en lo educativo. De este modo, de las textualidades emergen tres aspectos centrales que entendemos se derivan de concebir al mundo en constante movimiento. En primer lugar, el conocer en la diversidad y en la incertidumbre un mundo que coevoluciona con uno. Por tanto, se requiere abordar el aprendizaje en torno a la diversidad y la incertidumbre que rodea al estudiante. Así se vuelven centrales las nociones de cerca/lejos, que están siempre presente en una relación que tensiona. Cerca, asociado a la certidumbre, a los amigos y espacios ya conocidos y de cuyas prácticas se está apropiado, y lejos en la consciencia de lo que no se conoce, de lo incierto, de aquellas formas de hacer, actuar y 
conocer qué debo aprender, incorporar (Bauman 2007). Es una experiencia adaptativa constante que aleja de lo seguro y lleva a lo lejano, de este modo se requiere pasar a los nuevos modos de producción del conocimiento, concibiendo como un sujeto nómade, con capacidad de participación y experticia adaptativa (Andrade, 2005).

Un segundo elemento, es la construcción de sentido en torno a un mundo que evoluciona y es complejo. Es asumir el constante cambio y por tanto avanzar en la comprensión de la alta imbricación local y global que desafía al sujeto y por tanto a la educación. Se requiere, entonces, un cambio en el pensamiento y por ello en actividad para la construcción del estudiante (Flores, 2011). Es un entender que la complejidad no admite conclusiones, es fluir del ser, es conciencia y realidad (Andrade, 2005). Al mismo tiempo es camino, pensamiento, ciencia y contexto. Explicación adecuada a la realidad, considerándola caótica, diversa, compleja en si misma (Armijos, Hernández y Sánchez, 2017).

El tercer elemento destacado es la Construcción de una Conciencia múltiple que se imbrican para entender y actuar en este mundo incierto. Una consciencia sobre el aprendizaje a de ser constante, toda la vida, afectando al ethos cultural de modo de constituir una sociedad que siempre aprende (Flores, 2011); una conciencia ética, que comprende la extensa red de interdependencia en la cual todos somos responsables, tanto si lo sabemos cómo si no, tanto si nos gusta o no, tanto si responde a nuestra intención como si no de las miserias de los demás. En síntesis, se conforma un constructo que de los demás. (Bauman, 2012). Una consciencia global y particular, en cuanto debemos superar la sola preocupación por aquellos cercanos (sea cual sea el motivo) a nosotros comprendiendo la preocupación por todos. Una consciencia en constante construcción y deconstrucción de un pensamiento, un pensamiento que cobra conciencia de la conciencia y de la alteridad (Andrade, 2005). En síntesis, es un comprender la constante transformación, que cada uno ha de vivir para estar en un mundo en constante e interdependiente evolución.

De este modo, para la noción de aprendizaje se reconocen otros requerimientos (ver table 3) para una educación que prepare para vivir la incertidumbre, surge la necesidad de desarrollar un pensamiento dialógico, retroactivo, recursivo, hologramático, que aborda la condición de autonomía y dependencia constante, que asume la incertidumbre, y la transferencia y práctica del aprendizaje, en que la noción de diferencia este a la base de nuestro vivir (Armijos, Hernández y Sánchez, 2017). 
Tabla 3. Dimensiones y variables seleccionadas para "Aprender para"

\begin{tabular}{|l|l|}
\hline Dimensión & Variable \\
\hline \multirow{2}{*}{ Mundo en Constante Movimiento } & Conocer la diversidad \\
\cline { 2 - 2 } & Pensamiento Complejo \\
\cline { 2 - 2 } & Incertidumbre \\
\hline \multirow{2}{*}{ Desarrollo Colectivo } & Espacios de Aprendizaje \\
\cline { 2 - 2 } & Espacios de diálogo \\
\cline { 2 - 2 } Desarrollo del Pensamiento & Producción de conocimiento \\
\cline { 2 - 2 } & Uso de tecnología \\
\cline { 2 - 2 } & Analizar situaciones complejas \\
\hline \multirow{2}{*}{ Pensamiento Crítico } & Actuar en contextos complejos \\
\hline
\end{tabular}

Fuente: Elaboración propia (2019), a partir del análisis de contenido de la bibliografía.

\section{Conclusiones}

Esta fase del proyecto de investigación permitió avanzar en una resignificación de la noción de aprendizaje para la sociedad de la incertidumbre. A partir de esa revisión bibliográfica, se pudo caracterizar a la sociedad de la incertidumbre como una sociedad en la cual las seguridades del siglo XX son reemplazadas por el cambio e incertidumbre. Luego, la importancia de repensar aquello que entendemos por aprendizaje queda en evidencia al dejar la definición tradicional —en la que el aprendizaje se entiende como un concepto claramente definido- para avanzar en comprenderlo como una red conceptual, como un rizoma que imbrica diversas nociones de la acción humana. Algunas de las cuales destacamos en lo que sigue.

El aprendizaje como experiencia, es la vivencia que se transforma en conocimiento. Experiencia que es parte de nuestra existencia (Huxley, 1954), y por tanto, somos evolución, estereotipos y patrones que construyen el mundo que conocemos. Por su parte, John Dewey, precursor de la filosofía del aprendizaje experiencial, sostuvo que las experiencias pasadas tienen un impacto en la experiencia presente. conectaba el pasado, el presente y el futuro, reconociendo la importancia del recorrido biográfico en la construcción de aprendizajes. (Dewey 1964). La experiencia opera en el presente a partir de lo pasado, proyectando influjos positivos o negativos en el individuo hacia el futuro. Los principales elementos que la componen son el aprendizaje como producción, acción y proceso social, es decir siempre contextuado. 
El aprendizaje como producción es entendido como un proceso deductivo en el cual, los conocimientos generales permiten observar casos particulares y viceversa. Donde, el conocimiento es construido desde lo empírico. Así, desde esta perspectiva, los procesos educativos han de propiciar que el estudiantado pueda levantar hipótesis, supuestos o predicciones sobre lo que vive y observa que le permitan indagar en contextos específicos. Fortaleciendo de este modo una serie de aprendizajes traducidos en destrezas, habilidades y competencias, necesarias para enfrentar la incertidumbre. Aprendizajes que no necesariamente se traducen en destrezas, habilidades y competencias estandarizadas, sino en experiencias que le otorgan valor a su historia de vida o a su recorrido biográfico.

El aprendizaje es acción en una sociedad de la incertidumbre, ya que moviliza el conocimiento en pos de concretizarlo, lo pone en ejercicio en una actividad académica y productiva. Es una acción, dado que se sustenta en situaciones reales que ponen en contexto fenómenos sociales, culturales, económicos y artísticos. Logra impacto dado que la acción permite una perspectiva, en el mundo escolar, una perspectiva crítica, donde la argumentación es el fundamento para pasar de lo concreto a lo lógico deductivo, para elaborar posición crítica y mirada social, es así como el aprendiz adquiere habilidades superiores. En este punto el error como parte del aprendizaje toma relevancia nuevamente, dado que, en la acción y en la experiencia, dará cuenta de procesos que permitan fortalecer resiliencia y conocimiento para entender cómo aprendo y cómo soy capaz de incorporar conocimiento, situarlo, adaptarlo y hacerlo parte de mi quehacer.

La zona de desarrollo próximo (Vygotsky, 1931) toma un papel a considerar, dado que aquel que acompaña el aprendizaje proporcionará la profundidad, situándola y otorgándole las posibilidades de que lo que se aprende no quede en terrenos estancos y sea sustrato para levantar nuevas perspectivas, nuevos conocimientos o preguntas en torno a la acción vivida. Piaget (1971) sitúa como concepto la adaptación en general y la inteligencia en particular, otorgando un rol preponderante a la acción.

Como señalamos anteriormente el contexto hace que un aprendizaje sea pertinente y significativo, con ello las variables del contexto, del proceso social, incorporan elementos que le otorgan significado a ese aprendizaje. Lo social y económico imponen una manera de ver el mundo en una sociedad donde lo globalizado y el capitalismo imponen un abordaje en el mundo de la inmediatez. Es aquí donde las diferencias culturales impactan la disposición a aprender, ¿para qué sirve? o ¿no lo utilizaré nunca? son preguntas recurrentes. La procedencia, la historia personal, la historia de vida influyen y dan pie a condiciones para 
aprender, es en ciertos contextos "vulnerables" donde se profundizan y complejizan ciertos aprendizajes que no responden a lo llamado "socialmente aceptado" o "universalmente evaluado".

Esto nos alerta del desafío en la formación docente, pues, desde el lente de la sociedad líquida (Bauman 2007), se ha de considerar que el estudiantado lleva entre doce y catorce años de experiencia educativa que le ha permitido conformar un aprendizaje de lo escolar, que no siempre es coherente con nuevas perspectivas educativas.

Todos estos aspectos nos hacen pensar que hay que aprender para un constante desaprender, es lo que nos permite o permitirá avanzar hacia un cambio real y poder generar acciones de investigación y desarrollo que no solo avancen en generalizar estos resultados, sino que puedan impactar las resistencias de la Formación Inicial Docente con la urgencia que es necesaria en los actuales escenarios globales.

\section{Referencias}

Andrade, Raiza. (2005). Hacia una gnoseología del desaprendizaje dialógico cognoconsciente: Principios para desaprender en el contexto de la complejidad. Revista electrónica de investigación educativa, 7(2), 1-13. Universidad Autónoma de Baja California. México.

Arriagada, María, Ibañez, Paula y Godoy, Michal (2019). Aproximación a una noción de aprendizaje en la Sociedad de la Incertidumbre. En Edgar Serna (ed.), Revolución en la formación y la capacitación para el siglo XXI (2a. ed., Vol. 1) Medellín, Colombia: Instituto Antioqueño de Investigación.

Bauman, Zygmunt. (2005). Los retos de la educación en la modernidad líquida. Barcelona: Gedisa.

Bauman, Zygmunt. (2007). Miedo Líquido: La sociedad contemporánea y sus temores. Madrid: Paidós

Bonilla-García, Miguel. y López-Suárez, Ana. (2016). Ejemplificación del proceso metodológico de la teoría fundamentada. Cinta de Moebio, 57, 305-315. Recuperado de https://dx.doi.org/10.4067/S0717-554X2016000300006.

Bransford, John., Brown, Ann. y Cocking, Rodney. (2003). How People Learn. En Brain, Mind, Experience, and School. Washington, D.C.: National Academy Press.

Câmara, Helder Cavalcante., Fonseca, Ailton Siqueira de Souza., y Souza, Karlla Christine Araújo. (2014). Notas Introductorias Sobre A Formação Do Sujeito Em Zigmunt Bauman E Edgar Morin. Holos, 1, 290-297.

Chul Han Byung. (2019). La expulsión de lo distinto. España: Editorial Herder. 
Collingood-Selby, Elizabeth. (2018). Descripción General de los talleres de práctica del Departamento de Filosofía. Santiago: Universidad Metropolitana de Ciencias de la Educación.

De la Barrera, María Laura., y Donolo, Danilo. S. (2009). Neurociencias y su importancia en contextos de aprendizaje. Revista Digital Universitaria, 10(4), 1-18. México: UNAM

De Oliveira, Janaina., Henriksen, Danah., Castañeda, Linda., Marimon, Marta., Barberà, Elena., Monereo, Carles, ... Mishra, Punya. (2015). El panorama educativo de la era digital: prácticas comunicativas que (nos) impulsan hacia adelante. Colombia: Universidad de los Andes.

Dewey John. (1964) Naturaleza humana y conducta. México: Fondo de cultura económica.

Espín, Julia. (2002). El análisis de contenido: una técnica para explorar y sistematizar información. Revista de Educación, 4, 95-106. Universidad de Huelva, España.

Eteläpelto, Anneli., Vähäsantanen, Katja., Hökkä, Päivi., y Paloniemi, Susanna. (2013). What is agency? Conceptualizing professional agency at work. Educational Research Review, 10, 45-65.

Flores, José Humberto. (2011). Complejidad y educación. Revista Diálogos, 5(7), p. 23-34.

Gómez, Corona., Hernández, Mario., y Ramos Rodrigo. (2016). Principios epistemológicos para el proceso de la enseñanza-aprendizaje, según el pensamiento complejo de Edgar Morin. Pueblo Continente, 27(2), 471-479.

Guerrero, Susana. (2012). Guía para un uso igualitario y no sexista del lenguaje y de la imagen de la Universidad de Jaén. Jaén, España: La autora.

Hammerness, Karen. (2010). To seek, to strive, to find, and not to yield: A look at current conceptions of vision in education. In Second international handbook of educational change (pp. 1033-1048). Springer, Dordrecht.

Hammerness, Karen. (2010b). Indicadores de Calidad en la Formación Docente: Una mirada a las características de la Formación Docente desde una perspectiva internacional. Perspectivas Internacionales en Educación y Sociedad, 27, 239-277.

Huxley Aldous. (2013). Las puertas de la percepción. México: Editores mexicanos unidos.

Jiménez-Fontana, Rocio., García-González, Esther., Azcárate, Pilar., Navarrete, Antonio., y Cardeñoso, José Maria. (2016). La Teoría Fundamentada como estrategia de análisis de los datos: caracterización del proceso. CIAIQ2016, Portugal.

Lipponen, Lasse., y Kumpulainen, Kristiina. (2011). Acting as accountable authors: Creating interactional spaces for agency work in teacher education. Teaching and teacher education, 27(5), 812-819. 
Lasky, Sue. (2005). A sociocultural approach to understanding teacher identity, agency and professional vulnerability in a context of secondary school reform. Teaching and teacher education, 21(8), 899-916.

Ketelaar, Evelien, Beijaard, Douwe, Boshuizen, Henny, y Den Brok, Perry. (2012). Teachers' positioning towards an educational innovation in the light of ownership, sense-making and agency. Teaching and Teacher Education, 28(2), 273-282.

Morin, Edgar. (1999). Los siete saberes necesarios para la educación del futuro. París, Francia: Ed Santillana UNESCO

Piaget Jean. (2009). Psicología de la inteligencia (3a. ed.). Barcelona: Editorial de bolsillo.

Posner, Michael. y Rothbart, Mary. (2005) Influencing brain networks: implications for education. Trends in cognitive Sciences, 9(3), 99-103.

Pulido, Sergio. y Rodríguez, Javier. (2014). Manual básico de uso: Software NVivo, Versiones 9 y 10 . Bogotá: Universidad Nacional de Colombia. https://ucampus.umce.cl/umce/2019/2/EGB6011/1/material docente/

Reyes-Jedlicki, Leonora., Miranda, Christian., Santa Cruz, G. Eduardo., Cornejo Ch, Rodrigo., Núñez, Mauricio., Arévalo, Ana. y Hidalgo, Felipe. (2014). Subjetividades y saberes docentes en el sistema educativo chileno: un análisis desde las concepciones de formadores de profesores. Estudios pedagógicos, 40(ESPECIAL), 183-203.

Vasilachis de Gialdino, Irene. (2006). Estrategias de investigación cualitativa. En Irene Vasilachis de Gialdino (ed.), Estrategias de investigación cualitativa (pp. 23-64). Barcelona: Gedisa.

Vygotsky, Lev. (1931). Desarrollo de las funciones mnemónicas y mnemotécnicas. (Lev Vygotsky Obras Escogidas, Vol. 3, pp. 247-264). Moscú, Rusia: Pedagóguika. 
Revista indizada en

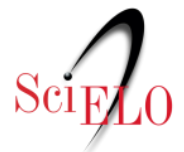

redalyc satindex

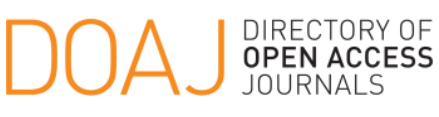

Distribuida en las bases de datos:

- Dialnet

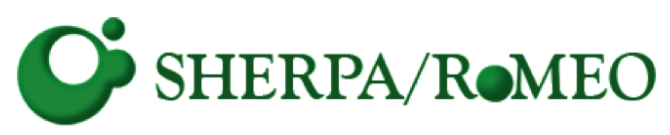

REDIB

Red Iberoamericana

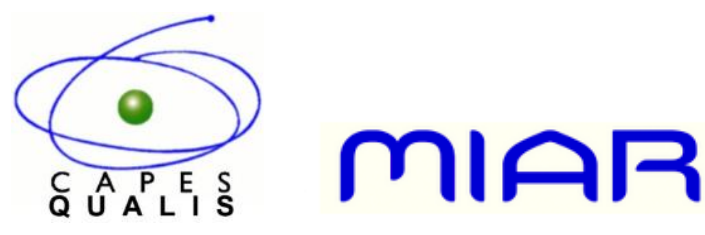

